\title{
Algoritmos Branch e Bound para o problema de sequenciamento em uma única máquina
}

\author{
Carlos E. Ferreira \\ Instituto de Matemática e Estatística, IME, USP \\ 05508-090, São Paulo, SP \\ E-mail: cef@ime.usp.br \\ Wellington D. Previero \\ UTFPR - Departamento de Matemática / Instituto de Matemática e Estatística, IME, USP \\ 86036-370, Londrina, PR / 05508-090, São Paulo, SP \\ E-mail: previero@utfpr.edu.br
}

\begin{abstract}
Resumo: Neste trabalho apresentamos o problema de minimização do makespan para o sequenciamento de tarefas em uma única máquina. Para este problema, cada tarefa está associada a um tempo de execução, um instante de disponibilidade de entrada no sistema e o tempo em que a tarefa fica no sistema após o seu processamento. Para a resolução do problema foram utilizados dois algoritmos baseados no método Branch e Bound e os resultados foram comparados. O primeiro algoritmo utiliza as estratégias de Carlier [2] e o segundo as de Grabowski, Nowicki e Zdrzalka [5]. O limitante inferior para o Branch e Bound é obtido através do Algoritmo Preemptivo de Jackson.
\end{abstract}

Palavras-chave: Sequenciamento em uma única máquina, escalonamento de tarefas

\section{Introdução}

O problema de sequenciamento em uma máquina (PSUM) consiste em ordenar um conjunto de tarefas para serem processadas em uma única máquina de modo a minimizar o tempo de conclusão da última tarefa a sair do sistema. Neste problema, cada tarefa está associada a um tempo de execução na máquina, um instante de disponibilidade de entrada no sistema e o tempo em que a tarefa fica no sistema após o seu processamento. Uma tarefa está finalizada assim que sair do sistema. Após o seu início, uma tarefa é processada até o seu término, sem interrupção.

O problema definido acima tem várias aplicações em sistemas de produção. Os resultados obtidos para o PSUM não só fornecem o conhecimento para o problema em si, como também podem proporcionar heurísticas a ambientes mais complexos, como o Job Shop ou o problema de sequenciamento de máquinas em paralelo. Para o Job Shop, podemos usar o PSUM para obter limitantes. Neste caso, uma máquina é escolhida e através da relaxação das restrições de outras máquinas obtemos limitantes para o problema. Isso nos ajuda a resolver uma das principais dificuldades do método Branch e Bound que é a ausência de bons limitantes inferiores para podar a árvore de enumeração tão cedo quanto o possível.

O PSUM é um problema Otimização Combinatória de difícil resolução. Garey e Johnson [4] e Lenstra, Kan e Brucker [9] mostraram que este problema é fortemente NP-difícil.

Carlier [2] desenvolveu um algoritmo Branch e Bound para resolver o problema de sequenciamento em uma máquina. As soluções factíveis são obtidas através do algoritmo de Schrage [12]. São apresentadas duas propostas de limitantes inferiores e as estratégias de ramificação são definidas a partir de escolha de uma tarefa específica e de um subconjunto de tarefas. Carlier e Pinson [3] desenvolveram um algoritmo Branch e Bound para o problema Job Shop baseado 
na resolução do PSUM. O algoritmo foi o primeiro a resolver um importante problema com 10 máquinas e 10 jobs proposto por Muth e Thompson [11].

Em Grabowski, Nowicki e Zdrzalka [5] é proposto um novo algoritmo Branch e Bound para resolver o PSUM. A abordagem utilizada é baseada em propriedades das tarefas pertencentes a um subconjunto de tarefas denominado bloco crítico. Estas propriedades definem a maneira como a alteração da ordem de uma tarefa em um sequenciamento pode influenciar na solução corrente. As propriedades definidas por Grabowski, Nowicki e Zdrzalka [5] também são utilizadas na resolução do problema de Job Shop [1], [6] e [3].

Liaw [10] apresenta um algoritmo Branch e Bound para o PSUM no qual em cada tarefa é atribuída uma penalização caso seja finalizada antes ou após um tempo limite. O limitante inferior de cada subproblema é obtido através da Relaxação Lagrangeana. O limite superior é obtido usando uma heurística de duas fases que combina uma regra de envio de prioridades com um processo de melhoria local.

O objetivo deste trabalho consiste em utilizar as estratégias de Carlier [2] e Grabowski, Nowicki e Zdrzalka [5] na resolução do PSUM e comparar os resultados obtidos.

\section{Descrição do Problema}

É dado um conjunto de $n$ tarefas independentes (jobs) indexadas $I=\{1, \ldots, n\}$ que devem ser processadas em uma única máquina. Para cada tarefa $i \in I$, sejam $r_{i} \geq 0$ o instante de disponibilidade da tarefa para a entrada no sistema, $p_{i}>0$ o tempo de processamento na máquina e $q_{i} \geq 0$ o tempo em que a tarefa fica no sistema após o seu processamento. Desejamos determinar para cada tarefa $i \in I$ o instante de início $t_{i}$ de seu processamento na máquina. Assim, $t_{i} \geq r_{i}$, para todo $i \in I$. Temos conhecimento de toda a informação sobre a tarefa antes da fase de sequenciamento (o problema é offline). O problema consiste em definir a ordem entre as tarefas que devem ser processadas na máquina de modo a minimizar o makespan, isto é, minimizar o tempo de conclusão da última tarefa a sair do sistema. Sejam $\pi=[\pi(1), \ldots, \pi(n)]$ o vetor permutação de $I$ e $\Pi$ o conjunto de todas as permutações. Sendo $C_{i}=t_{i}+p_{i}$ o tempo de conclusão da tarefa $i$, o problema consiste em encontrar a permutação $\pi \in \Pi$ que minimiza $C_{\text {Max }}(\pi)=\operatorname{Max}_{1 \leq i \leq n}\left(C_{i}+q_{i}\right)$, para todo $\pi \in \Pi$. Este problema é denotado por $1\left|r_{i}, q_{i}\right| C_{\text {Max }}$.

Dado um vetor permutação $\pi$, podemos associar a ordem de sequenciamento das tarefas de $\pi$ a um grafo $G=(V, A)$. O conjunto $V$ de vértices representa as tarefas $i \in I$, além das tarefas fictícias $O$ e $F$, que descrevem o início e o término do processamento, respectivamente. $\mathrm{O}$ conjunto $A$ de arcos é composto por três conjuntos: $A=U_{1} \cup U_{2} \cup U_{3}$, tal que $U_{1}=\{(O, i), i \in I\}$, $U_{2}=\{(i, F), \quad i \in I\}$ e $U_{3}=\{(i, j)$, se $i$ precede $j$ no sequenciamento em $\Pi\}$. A cada arco $(O, i) \in U_{1}$ é atribuído o valor $r_{i}$ e para cada arco $(i, F) \in U_{2}$ é atribuído o valor $q_{i}+p_{i}$. Além disso, para cada arco $(i, j) \in U_{3}$ é atribuído o valor $p_{i}$.

$\mathrm{O}$ instante de processamento $t$ da tarefa corresponde ao valor do maior caminho ponderado entre o nó $O$ e o nó $i$. Logo $t_{F}$ corresponde ao valor do makespan e

$$
C_{M a x}(\pi)=r_{\pi\left(i_{1}\right)}+\sum_{i=\pi\left(i_{1}\right)}^{\pi\left(i_{2}\right)} p_{i}+q_{\pi\left(i_{2}\right)}
$$

para algum par $\left(i_{1}, i_{2}\right)$ com $1 \leq i_{1} \leq i_{2} \leq n$ e $\pi\left(i_{1}\right), \pi\left(i_{2}\right) \in I$. A sequência $\left[\pi\left(i_{1}\right), \pi\left(i_{1}+\right.\right.$ $\left.1), \ldots, \pi\left(i_{2}\right)\right]$ de tarefas sucessivas de $\pi$ é denominada de bloco crítico de $\pi$ e será denotado por $B_{\pi}$. As tarefas $\pi\left(i_{1}\right)$ e $\pi\left(i_{2}\right)$ representam a primeira e a última tarefa do bloco, respectivamente.

\section{Método Branch e Bound}

Nesta seção apresentaremos dois procedimentos para a resolução do PSUM através do método Branch e Bound. No primeiro método é utilizada a proposta de Carlier [2] (Algoritmo 1) e no 
segundo a de Grabowski, Nowicki e Zdrzalka [2] (Algoritmo 2). Para cada método, definiremos os procedimentos para o cálculo dos limitantes superior e inferior e a estratégia de ramificação.

\subsection{Algoritmo 1}

O limitante superior para o problema é determinado através do Algoritmo de Schrage. O algoritmo em consiste em dividir, em cada iteração, o conjunto de tarefas $I$ em dois conjuntos: o conjunto de tarefas sequenciadas $(S)$ e o conjunto de tarefas não sequenciadas $(N)$. Inicialmente temos que $S=\emptyset$ e $N=I$ e $t$ é inicializado com $M i n_{i \in N} r_{i}$. No tempo $t$, dentre as tarefas $i \in N$ tais que $r_{i} \leq t$, selecionamos a que possui o maior valor de $q_{i}$. A tarefa $i$ selecionada é removida do conjunto $N$ e adicionada ao conjunto $S$. O valor de $t$ é atualizado para $t=$ $\operatorname{Max}\left(t_{i}+p_{i}, \operatorname{Min}_{i \in N} r_{i}\right)$. O algoritmo é finalizado quando todas as tarefas do conjunto $N$ são sequenciadas, isto é, $N=\emptyset$. Na próxima proposição mostramos como obter o limitante inferior para o valor do makespan.

Proposição 1. Para todo $I_{t} \subseteq I$, temos que

$$
h\left(I_{t}\right)=\operatorname{Min}_{i \in I_{t}} r_{i}+\sum_{i \in I_{t}} p_{i}+\operatorname{Min}_{i \in I_{t}} q_{i}
$$

é um limitante inferior para o valor ótimo do makespan.

Limitantes inferiores mais justos que o apresentado na Proposição 1 podem ser obtidos, por exemplo, através do Algoritmo Preemptivo de Jackson [7]. Neste algoritmo um escalonamento das tarefas é obtido permitindo preempção, ou seja, uma tarefa pode ser interrompida em qualquer instante. A ideia é então, seguir a mesma ordenação proposta no algoritmo de Scharage (maior valor de $q_{i}$ entre as tarefas disponíveis para execução) interrompendo a execução de uma tarefa sempre que outra tarefa $j$ tiver $q_{j}>q_{i}$. Desta forma, minimizamos o tempo ocioso da máquina. Obviamente isso é uma relaxação do nosso problema (em que a preempção não é permitida), e portanto o valor do makespan obtido é um limitante inferior para o makespan ótimo quando preempção não é permitida. $[2]$.

A complexidade do Algoritmo de Schrage e do Algoritmo Preemptivo de Jackson é $O(n \log n)$

Proposição 2. O makespan do Algoritmo Preemptivo de Jackson é um limitante inferior para o makespan ótimo do problema de sequenciamento de uma única máquina. Além disso, o seu valor é igual a $\operatorname{Max}_{I_{t} \subset I} h\left(I_{t}\right)$.

O próximo teorema implica que se o sequenciamento obtido pelo algoritmo de Scharage não for ótimo, então está "próximo" do ótimo, e para melhorá-lo, uma tarefa específica $\pi(k)$ tem que ser escolhida.

Proposição 3. Sejam $\pi$ e $L$ o sequenciamento e o makespan obtido pelo algoritmo de Schrage para as tarefas do conjunto $I$, respectivamente. Se o sequenciamento $\pi$ é ótimo, então existe um conjunto $J$ tal que $h(J)=L$. Caso contrário, existe uma tarefa crítica $\pi(k)$ e um conjunto crítico $J$ tal que $h(J)=\operatorname{Min}_{\pi(j) \in J} r_{\pi(j)}+\sum_{\pi(j) \in J} p_{\pi(j)}+\operatorname{Min}_{\pi(j) \in J} q_{\pi(j)}>L-p_{\pi(k)}$.

A tarefa $\pi(k)$ e o conjunto $J$ são obtidos da seguinte forma. Sejam $\pi$ e $L$ o sequenciamento e o makespan obtido pelo algoritmo de Schrage, respectivamente. Suponha que $B_{\pi}$ seja o bloco crítico definido por $\pi$ com um número máximo de tarefas. Se $h\left(B_{\pi}\right)=L$, então o sequenciamento é ótimo. Logo para todos $\pi(i), \pi(j) \in B_{\pi}$, com $i_{1} \leq i<j \leq i_{2}$, temos que $q_{\pi(i)} \geq q_{\pi(j)}$. Se $\pi$ não é ótimo, então existe $i<i_{2}$ tal que $q_{\pi(i)}<q_{\pi\left(i_{2}\right)}$. A tarefa $\pi(k)$ é escolhida como sendo a última tarefa de $B_{\pi}$ tal que $q_{\pi(k)}<q_{\pi\left(i_{2}\right)}$ e o conjunto $J$ é definido pro $J=\left\{\pi(k+1), \ldots, \pi\left(i_{2}\right)\right\}$. O corolário seguinte define a estratégia de ramificação utilizado pelo Algoritmo 1. 
Corolário 1. Se $h(J)>L-p_{\pi(k)}$, então a distância entre o makespan ótimo e o makespan do sequenciamento do Algoritmo de Schrage é menor que $p_{\pi(k)}$. Além disso, no sequenciamento ótimo, ou $\pi(k)$ será processado antes que todas as tarefas de $J$ ou $\pi(k)$ será processado após todas as tarefas de $J$.

A cada nó do algoritmo Branch e Bound é associado um PSUM, que fornece um limitante inferior $f_{\text {Min }}$. O Algoritmo Preemptivo de Jackson é utilizado para o cálculo do limitante inferior. O limitante superior $f_{\text {Max }}$ é o atualizado por $f_{\text {Max }}=\operatorname{Min}\left\{f_{\text {Max }}, L\right\}$, onde $L$ é o makespan do sequenciamento produzido pelo algoritmo de Schrage no nó atual. O nó com o menor limitante inferior na árvore de busca é selecionado e aplica-se o algoritmo de Schrage para gerar o sequenciamento das tarefas. Se não for possível determinar uma tarefa crítica $\pi(k)$ para o sequenciamento obtido, então o sequenciamento é ótimo. Caso contrário, temos dois novos problemas. O primeiro problema em que a tarefa $\pi(k)$ é sequenciada antes de todas as tarefas do conjunto crítico $J$ e o segundo problema em que a tarefa $\pi(k)$ é forçada a ser sequenciada após todas as tarefas do conjunto crítico $J$. Se $\pi(k)$ é processado antes de todas as tarefas de $J$, o valor de $q_{\pi(k)}$ pode ser atualizado para $q_{\pi(k)}=\operatorname{Max}\left(q_{\pi(k)}, \sum_{\pi(j) \in J} p_{\pi(j)}+\operatorname{Min}_{\pi(j) \in J} q_{\pi(j)}\right)$. Para que a tarefa $\pi(k)$ seja processada após todas as tarefas de $J$, podemos fazer que $r_{\pi(k)}=$ $\operatorname{Max}\left(r_{\pi(k)}, \operatorname{Min}_{\pi(j) \in J} r_{\pi(j)}+\sum_{\pi(j) \in J} p_{\pi(j)}\right)$. Estas alterações ficam fixas para o nó e todos os seus descendentes. Os novos nós serão acrescentados na árvore somente quando o limitante inferior for menor que o limitante superior.

\subsection{Algoritmo 2}

Se $\pi$ representa uma permutação das tarefas de $I$, então o valor $C_{\text {Max }}(\pi)$ define um limitante superior para o makespan do sequenciamento ótimo. O limitante inferior utilizado pelo Algoritmo 2 é o descrito pela Proposição 2. O próximo teorema define a estratégia de ramificação do método Branch e Bound.

Proposição 4. Sejam $\pi$ uma permutação de tarefas do conjunto $I$ e $B_{\pi}$ o conjunto de tarefas do bloco crítico da permutação $\pi$. Se $\beta$ é qualquer permutação de $I$ tal que $C_{\text {Max }}(\beta)<C_{\text {Max }}(\pi)$, então existe uma tarefa $\pi(k) \in B_{\pi}-\left\{\pi\left(i_{1}\right)\right\}$ (ou $\pi(k) \in B_{\pi}-\left\{\pi\left(i_{2}\right)\right\}$ ) e na permutação $\beta$ a tarefa $\pi(k)$ precede (ou sucede) todas as tarefas do conjunto $B_{\pi}-\{\pi(k)\}$.

Através da Proposição 4 podemos construir, a partir de um sequenciamento $\pi$, um possível sequenciamento de melhor makespan analisando dois subproblemas para as tarefas de seu bloco crítico: no primeiro subproblema a tarefa é executada antes (posição $i_{1}$ ) de todas as tarefas do bloco crítico, e no segundo depois de todas as outras tarefas do bloco (posição $i_{2}$ ).

As tarefas a serem deslocadas para a posição $i_{1}$ serão tomadas do conjunto $T_{b}=\{\pi(j) \in$ $\left.B_{\pi} ; \Delta_{b}(\pi(j))<0\right\}$, tal que $\Delta_{b}(\pi(j))=p_{\pi(j)}-p_{\pi\left(i_{1}\right)}$; as tarefas deslocadas para a posição $i_{2}$ serão tomadas do conjunto $T_{a}=\left\{\pi(j) \in B_{\pi} ; \Delta_{a}(\pi(j))<0\right\}$, com $\Delta_{a}(\pi(j))=q_{\pi(j)}-q_{\pi\left(i_{2}\right)}$.

Seja $\beta$ o sequenciamento obtido a partir de $\pi$ através do deslocamento da tarefa $\pi(k) \in$ $T_{b}\left(\pi(k) \in T_{a}\right)$. Assim, todos os descendentes de $\beta$ devem ter a propriedade de que a tarefa $\pi(k)$ seja processada antes (após) de todas as tarefas do conjunto $T_{b}^{\prime}=T_{b} \cup\left\{\pi\left(i_{1}\right)\right\}$ $\left(T_{a}^{\prime}=T_{a} \cup\left\{\pi\left(i_{2}\right)\right\}\right)$. Logo, cada nó da árvore está associado a um conjunto de restrições de prioridades. Seja $R_{\pi}$ o conjunto de restrições de prioridades associado a $\pi$. Para que as restrições de $R_{\pi}$ sejam respeitadas para os novos descendentes de $\pi$, o conjunto $T_{b}$ é redefinido para $T_{b}=\left\{\pi(j) \in B_{\pi} ; \Delta_{b}(\pi(j))<0\right.$ e $\pi(j)$ não é executada após a tarefa $\pi(k)$ em $R_{\pi}, k=$ $\left.i_{1}, \ldots, j-1\right\}$. De forma análoga, o conjunto $T_{a}$ é redefinido por $T_{a}=\left\{\pi(j) \in B_{\pi} ; \Delta_{a}(\pi(j))<\right.$ 0 e $\pi(j)$ não é executada antes da tarefa $\pi(k)$ em $\left.R_{\pi}, k=j+1, \ldots, k\right\}$.

Quando a tarefa $\pi(k)$ é deslocada para a posição $i_{1}$ os valores de $r_{i}$ e $q_{i}$ são atualizados para $r_{\pi(j)}=\operatorname{Max}\left(r_{\pi(j)}, r_{\pi(k)}+p_{\pi(k)}\right)$, para todo $\pi(j) \in T_{b}^{\prime}, \pi(j) \neq \pi(k)$, e $q_{\pi(k)}=\operatorname{Max}\left(q_{\pi(k)}\right.$, $\left.\sum_{\pi(j) \in T_{b}^{\prime}} p_{\pi(j)}-p_{\pi(k)}+\operatorname{Min}_{\pi(j) \in T_{b}^{\prime} \backslash\{\pi(k)\}} q_{\pi(j)}\right)$. 
Agora, uma vez que a tarefa $\pi(k)$ é deslocada para a posição $i_{2}$, os valores de $r_{i}$ e $q_{i}$ são fixados em $r_{\pi(k)}=\operatorname{Max}\left(r_{\pi(k)}, \sum_{\pi(j) \in T_{a}^{\prime}} p_{\pi(j)}-p_{\pi(k)}+\operatorname{Min}_{\pi(j) \in T_{a}^{\prime} \backslash\{\pi(k)\}} r_{\pi(j)}\right)$ e $q_{\pi(j)}=$ $\operatorname{Max}\left(q_{\pi(j)}, p_{\pi(k)}+q_{\pi(k)}\right)$ para todo $\pi(j) \in T_{a}^{\prime}$ e $\pi(j) \neq \pi(k)$.

Para iniciar o método Branch e Bound, uma permutação inicial $\pi$ é obtida através do Algoritmo de Schrage. Os nós descendentes da permutação $\pi$ são obtidos através dos deslocamentos das tarefas nos conjuntos $T_{b}$ e $T_{a}$ e adicionados a árvore de pesquisa. Cada nó descendente de $\pi$ representa uma nova permutação de tarefas de $I$. O nó com o menor limitante inferior na árvore de busca é selecionado e novos possíveis descendentes são gerados. Grabowski, Nowicki e Zdrzalka [5] utilizam a estratégia de Backtracking para a resolução do PSUM.

\section{Experimentos Computacionais}

O algoritmo Branch e Bound foi implementado em linguagem Java versão 1.7 utilizando Eclipse IDE versão Juno Service Release 2. Os testes foram realizados em um computador Acer Intel Core i5/2.53 GHz e 4 GB de memória ram. Os problemas testes em Carlier [2] e Grabowski, Nowicki e Zdrzalka [5] foram gerados através da metodologia descrita por Lentra [8]. Desta forma utilizamos o mesmo procedimento para gerar os problemas testes. Definido o valor de $n$ para o número de tarefas do PSUM, os valores $r_{i}, q_{i}$ e $p_{i}$ foram gerados a partir de uma distribuição uniforme entre 1 e $r_{\text {Max }}, 1$ e $q_{\text {Max }}$ e 1 e $d_{\text {Max }}$, respectivamente. Os valores $r_{\text {Max }}, q_{\text {Max }}$ e $d_{\text {Max }}$ foram definidos por $r_{\text {Max }}=50 R, q_{\text {Max }}=50 Q$ e $d_{\text {Max }}=50$, com $R, Q=\{0,5 ; 2 ; 0,5 n, 2 n\}$. Para cada valor de $n=\{20,40,80,150,200\}, 80$ testes foram gerados, sendo 5 testes para cada combinação dos parâmetros $R$ e $Q$. O limitante inferior descrito pela Proposição 2 foi utilizado em ambos os algoritmos. As tabelas 1 e 2 exibem os resultados computacionais. O tempo máximo, mínimo e a média para a execução de cada algoritmo foi calculado para cada valor de $n$. A unidade de tempo utilizada é o milissegundo $(\mathrm{ms})$. Calculamos também o máximo e a média do número de nós gerados por cada algoritmo, além da quantidade de testes na qual a solução ótima foi obtida no primeiro nó da árvore.

\begin{tabular}{|c|c|c|c|c|c|c|}
\hline \multirow[t]{3}{*}{$n$} & \multicolumn{6}{|c|}{ Algoritmo 1} \\
\hline & \multicolumn{2}{|c|}{ Número Nós } & \multicolumn{3}{|c|}{ Tempo Execução } & \multirow[t]{2}{*}{ Solução no Primeiro Nó } \\
\hline & Média & Max & Min & Média & $\operatorname{Max}$ & \\
\hline 20 & 6,05 & 90 & 0 & 1,17 & 16 & 51 \\
\hline 40 & 14,57 & 263 & 0 & 8,96 & 124 & 50 \\
\hline 80 & 23,17 & 761 & 0 & 67,70 & 1840 & 54 \\
\hline 150 & 10,44 & 136 & 15 & 161,55 & 1670 & 53 \\
\hline 200 & 13,66 & 250 & 31 & 498,59 & 10640 & 56 \\
\hline
\end{tabular}

Tabela 1: Resultados Computacionais do Algoritmo 1

\begin{tabular}{|c|c|c|c|c|c|c|}
\hline \multirow[t]{3}{*}{$n$} & \multicolumn{6}{|c|}{ Algoritmo 2} \\
\hline & \multicolumn{2}{|c|}{ Número Nós } & \multicolumn{3}{|c|}{ Tempo Execução } & \multirow[t]{2}{*}{ Solução no Primeiro Nó } \\
\hline & Média & Max & Min & Média & Max & \\
\hline 20 & 3,35 & 86 & 0 & 1,25 & 30 & 59 \\
\hline 40 & 3,75 & 40 & 0 & 2,33 & 31 & 57 \\
\hline 80 & 5,70 & 143 & 0 & 8,18 & 125 & 59 \\
\hline 150 & 3,47 & 55 & 15 & 31,40 & 358 & 64 \\
\hline 200 & 4,82 & 77 & 31 & 72,81 & 406 & 61 \\
\hline
\end{tabular}

Tabela 2: Resultados Computacionais do Algoritmo 2

O número de nós produzidos pelo Algoritmo 1 variou entre 1 e 761 e no Algoritmo 2, o 
número de nós alterou entre 1 e 143 . Em todos os valores de $n$, a média de nós gerados pelo Algoritmo 2 foi inferior a do Algoritmo 1.

A solução ótima foi obtida no primeiro nó da árvore para 0,66\% dos testes no Algoritmo 1 e $0,75 \%$ no Algoritmo 2. Isto indica que ambos os Algoritmos reconheceram rapidamente, como ótimo, o sequenciamento obtido pelo Algoritmo de Schrage.

O tempo de execução do Algoritmo 1 para resolver os problemas testes variou entre 0 e 10,64 segundos. Para o Algoritmo 2, a variação de tempo ficou 0 e 0,406 segundo. Exceto para o caso de $n=20$, a média de tempo para o Algoritmo 2 foi inferior a do Algoritmo 1. A diferença significativa entre os tempos nos dois Algoritmos se deve a fato de que o Algoritmo 1 gerou, em média, mais nós na árvore de busca e a cada nó gerado um tempo adicional foi incorporado em decorrência da execução do Algoritmo de Schrage.

\section{Conclusão}

Neste trabalho comparamos o desempenho computacional de dois algoritmos para o problema de minimização do makespan no sequenciamento de tarefas em uma única máquina. Cada tarefa tem um tempo de processamento, um tempo de entrada no sistema e um tempo em que a tarefa fica no sistema após a sua conclusão. Para resolver o problema, utilizamos o método Branch e Bound e as estratégias utilizadas por Carlier [2] (Algoritmo 1) e Grabowski, Nowicki e Zdrzalka [5] (Algoritmo 2). Ambos os algoritmos determinaram a solução ótima para os 400 problemas testes. Foram analisados para cada algoritmo o número de nós gerados na árvore de busca e o tempo de processamento. O Algoritmo 2 apresentou os melhores resultados, tanto para o número de nós, quanto para o tempo de processamento. Em ambos os Algoritmos observou-se a repetição de muitos sequenciamentos na árvore de busca, além de muitos sequenciamentos com o mesmo limitante inferior. Uma possibilidade para melhoria do algoritmo seria a utilização do procedimento de Backtracking na árvore de busca e de novas formas de atualização de $r_{i}$ e $q_{i}$.

\section{Referências}

[1] P. Brucker, B. Jurisch, B. Sievers, A branch and bound algorithm for the job-shop scheduling problem, Discrete Applied Mathematics, 49, (1994), 107-127.

[2] J. Carlier, The one-machine sequencing problem, European Journal of Operational Research, $11,(1982), 42-47$.

[3] J. Carlier, E. Pinson, An algorithm for solving the job-shop problem, Management science, 35, (1989), 164-176.

[4] M. R. Garey, D. S. Johnson, "Computers and Intractability: A Guide to the Theory of NP-Completeness", Freeman, New York, 1979.

[5] J. Grabowski, E. Nowicki, S. Zdrzalka, A block approach for single-machine scheduling with release dates and due dates, European Journal of Operational Research, 26, (1986), 278-285.

[6] J. Grabowski, J. Pempera, The permutation flow shop problem with blocking. A tabu search approach, Omega, 35, (2007), 302-311.

[7] J. R. Jackson, Scheduling a production line to minimize maximum tardiness, Management Science Research, 43, (1955).

[8] J. K. Lentra, "Sequencing by enumerative methods", Mathematisch Centrum Amsterdam, (1976).

[9] J. K. Lenstra, A. R. Kan, P. Brucker, Complexity of machine scheduling problems, Econometric Institute of the Erasmus University, (1977). 
[10] C. F. Liaw, A branch-and-bound algorithm for the single machine earliness and tardiness scheduling problem, Computers and Operations Research, 26, (1999), 679-693.

[11] J. F. Muth, G. L. Thompson, "Industrial Scheduling", Prentice Hall, New Jersey, 1963.

[12] L. Schrage, Solving resource-constrained network problems by implicit enumeration nonpreemptive case, Operations Research , 18, (1970), 263-278. 